\title{
Development of Organic Gas Sensor Using Quartz Crystal Microbalance Coated with Plasma-polymerized Films
}

\author{
Hidenobu Aizawa, ${ }^{1 *}$ Shin-ichi Kusakari, ${ }^{1,2}$ Kazunori Yamada, ${ }^{2}$ \\ Kazutoshi Noda, ${ }^{1}$ and Hiroshi Habe ${ }^{1}$ \\ ${ }^{1}$ Environmental Management Research Institute, National Institute of Advanced Industrial Science \\ and Technology (AIST), 16-1 Onogawa, Tsukuba, Ibaraki 305-8569, Japan \\ ${ }^{2}$ Department of Applied Molecular Chemistry, College of Industrial Technology, Nihon University, \\ 1-2-1 Izumi-cho, Narashino, Chiba 275-8575, Japan
}

(Received September 27, 2019; accepted January 14, 2020)

Keywords: quartz crystal microbalance (QCM), plasma polymerization, thin film, gas sensor, volatile organic compound (VOC)

In this study, we developed a gas detection membrane that can selectively sense volatile organic compounds used in the industry and research. 4-(Chloromethyl) styrene (CMS) and 4-vinylbenzoic acid (VBA) films were coated on a quartz crystal microbalance (QCM) using plasma polymerization. The response sensitivity (RS) to each organic gas in the plasmapolymerized (pp) film was calculated and evaluated from the amount of frequency shift in the organic gas adsorption/desorption of the QCM. In pp-CMS coated on the QCM, dichloromethane was adsorbed more than in other pp-films, possibly because pp-CMS had a higher affinity with chloro groups of dichloromethane. By contrast, pp-VBA had a high RS to alcohol-based solvents containing hydroxy groups, especially methanol. From the results, the gas selectivity of the pp films was suggested to depend on their functional groups.

\section{Introduction}

In industry and research facilities, many organic solvents are used in manufacturing processes and experiments. ${ }^{(1)}$ These organic solvents are designated as volatile organic compounds (VOCs). ${ }^{(2-4)}$ VOCs are a concern because they give rise to the generation of photochemical oxidants and suspended particulate matter that damage humans and plants. ${ }^{(5)}$ Also, VOCs have been reported to cause health problems such as carcinogenesis after longterm exposure; thus, countermeasures are required. ${ }^{(6-12)}$ If we can constantly monitor the types and concentrations of VOCs used in manufacturing processes and laboratories, we can maintain a safe work environment. In this study, polymer thin films that can detect VOCs were formed by plasma polymerization, and their gas adsorption characteristics and desorption were investigated using a quartz crystal microbalance (QCM). ${ }^{(13)}$

The QCM is an element in which metal electrodes are attached to both sides of a quartz plate known as a piezoelectric body. In the QCM, the frequency decreases proportionally to the *Corresponding author: e-mail: hide-aizawa@aist.go.jp https://doi.org/10.18494/SAM.2020.2637 
increase in mass owing to the substance adsorbed on the electrode surface. The mass sensitivity is calculated from the Sauerbrey ${ }^{(14)}$ equation

$$
\Delta f=\frac{-2 f_{0}^{2} \Delta M}{A \sqrt{\rho \mu}} .
$$

Here, $f_{0}$ is the fundamental frequency $(\mathrm{Hz}), A$ is the electrode area $\left(\mathrm{cm}^{2}\right), \rho$ is the crystal density $\left(\mathrm{g} / \mathrm{cm}^{3}\right)$, and $\mu$ is the shear modulus $\left(\mathrm{g} \cdot \mathrm{cm} \cdot \mathrm{s}^{-2}\right)$ of the crystal. The mass sensitivity of the ATcut $9 \mathrm{MHz}$ of the QCM used in this study is about $1\left(\mathrm{ng} / \mathrm{cm}^{2} \mathrm{~Hz}\right)$. In this manner, a very small amount of mass change can be measured, so it is used for research on film thickness in meters and chemical sensors. ${ }^{(15)}$

To induce QCM gas selectivity, a polymer thin film is coated on the QCM by plasma polymerization. Unlike radical or ion polymerization, plasma polymerization can be performed by a dry process that does not use a liquid. In plasma polymerization, a thin film can be obtained by a one-step reaction using a thermal nonequilibrium state caused by high-frequency discharge in a vacuum reaction vessel mainly decompressed. ${ }^{(16)}$ The monomer introduced into the plasma space by gas or sublimation is excited in the plasma space and is turned into plasma including reactive active species such as fast electrons, cations, and radicals. In this research, when performing plasma polymerization, the QCM was placed on the lower electrode to polymerize a liquid monomer or attached to the upper electrode with tape to coat the polymer films.

The principle of gas adsorption is that the functional groups present on the surface of a plasma-polymerized (pp) film are combined with organic gas to adsorb them. The gas adsorption characteristics of each polymerized film are measured and evaluated from the frequency change of the QCM, which is caused by the minute mass change of the polymerized film. ${ }^{(17-22)}$

\section{Materials and Methods}

\subsection{Materials and equipment}

4-(Chloromethyl) styrene and 4-vinylbenzoic acid purchased from Tokyo Chemical Industry Co., Ltd. (Tokyo, Japan) were used as monomers without further purification. Methanol, ethanol, acetone, isopropyl alcohol (2-propanol), chloroform, and dichloromethane were purchased from FUJIFILM Wako Pure Chemical Corporation (Osaka, Japan). The AT-cut $9 \mathrm{MHz} \mathrm{Au}$ electrodes purchased from Nihon Dempa Kogyo Co., Ltd. (Tokyo, Japan) were used for the QCM. Prior to coating the QCM, the electrode surface of the QCM was cleaned using the plasma polymerization equipment Plasma Basic Kit Model BP-1 (Samco Inc., Kyoto, Japan) for $60 \mathrm{~s}$ with Ar plasma at a radio frequency (RF) power of $100 \mathrm{~W}$ and an inner bell jar pressure of $100 \mathrm{~Pa}$. After Ar plasma cleaning, the frequency of each QCM was measured using a 16-channel QCM sensor unit fabricated by AIST. 


\subsection{Plasma polymerization}

The liquid monomer 4-(chloromethyl) styrene (CMS) was vaporized by adjusting the temperature to $50{ }^{\circ} \mathrm{C}$ using a water bath. Four QCMs were arranged on the lower electrode as shown in Fig. 1(a). After replacing the inside of the bell jar with Ar gas and supplying monomer gas at a pressure of $5 \mathrm{~Pa}$, a pp-CMS film was coated on the QCM for 1 min at RF powers of 90 , 110, 130, and $150 \mathrm{~W}$. By contrast, a sublimation method was used to introduce 4-vinylbenzoic acid (VBA), which is solid at room temperature, into the plasma space. As shown in Figs. 1(b) and 1(c), the QCM was fixed to the upper electrode, VBA was placed in an alumina crucible (The Nilaco Corporation, Tokyo, Japan), and the space between the QCM and the crucible was blocked with a shutter. After replacing the inside of the bell jar with Ar gas, the crucible was heated at a DC of $1.2 \mathrm{~V}$ and a current of $20 \mathrm{~A}$ to sublimate the monomer. When the monomer sublimation was stabilized, the shutter was opened, and a pp-VBA film was coated on the QCM for $1 \mathrm{~min}$ at RF powers of 90, 110, 130, and $150 \mathrm{~W}$. Films were formed on the four QCMs for each monomer and each RF power. The frequency of the QCM after pp film coating was measured again with a 16-channel QCM sensor unit, and the film thickness was calculated from the amount of frequency change before and after the coating. The thickness of pp films was calculated from the frequency shift of the QCM as

$$
t=-\frac{\Delta f}{2.26 \times 10^{-7} \cdot f_{0}^{2} \cdot \rho_{m}}
$$

where $t$ is the thickness of pp films, $\Delta f$ is the frequency shift of the QCM caused by the attached mass of pp films, $f_{0}$ is the fundamental frequency, and $\rho_{m}$ is the density of a monomer.

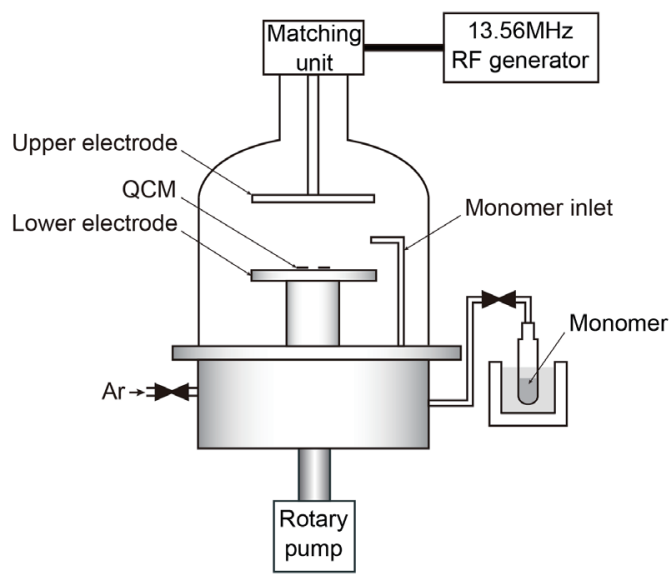

(a)

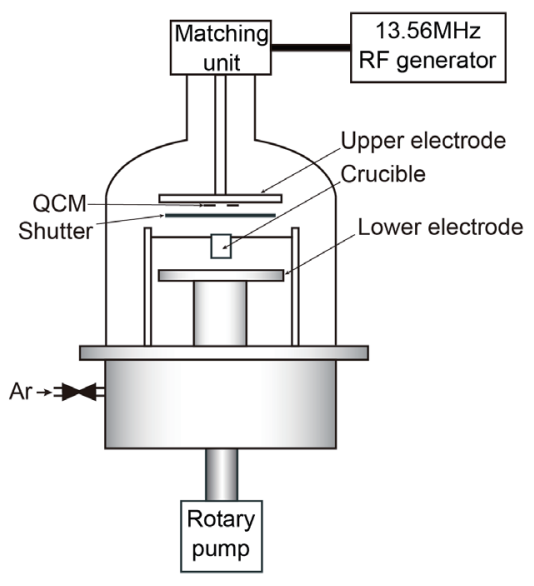

(b)

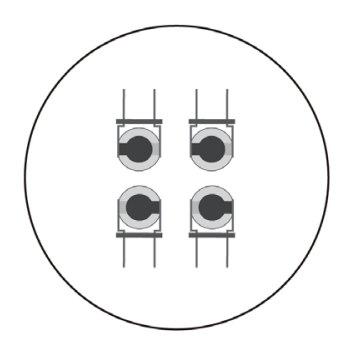

(c)

Fig. 1. Schematic diagrams of plasma polymerization equipment. Polymerizing with liquid monomer (CMS) (a), polymerizing with solid monomer (VBA) (b), and (c) positions of four QCMs set on upper and lower electrodes. 


\subsection{Fourier transform infrared (FT-IR) spectroscopy}

The FT-IR spectra of the generated films were obtained using a FT/IR-610 (Jasco, Inc., Tokyo, Japan) at a resolution of $2 \mathrm{~cm}^{-1}$ while the instruments were purged with $\mathrm{N}_{2}$ gas. Each spectrum was averaged over 128 scans. The monomers of CMS and VBA were measured by FT-IR spectroscopy with the liquid membrane and $\mathrm{KBr}$ tablet methods, respectively. The measurement was performed by transmission. A pp film was coated on $\mathrm{KBr}$ in the same manner as that on the QCM. For FT-IR spectroscopy, which requires a film thickness of $1 \mu \mathrm{m}$, a pp film was coated on $\mathrm{KBr}$ with a polymerization time of $2 \mathrm{~min}$.

\subsection{SEM}

The QCM electrode surfaces were observed by SEM (JSM-6010A, JEOL Ltd., Tokyo, Japan) to investigate the shape of the pp film deposited at each RF power. Measurements were performed at an acceleration voltage of $10 \mathrm{kV}$ and a magnification of $1000 \times$; QCMs were immediately observed after Pt-Pd coating.

\subsection{Sample gas adsorption and response sensitivity (RS) calculation}

The QCM coated with pp-CMS and pp-VBA films at 90, 110, 130, and $150 \mathrm{~W}$ was attached to a 16-channel sensor unit installed in a thermostatic chamber maintained at $25{ }^{\circ} \mathrm{C}$ and sealed in a $520 \mathrm{ml}$ measurement vessel as shown in Fig. 2. Then, the oscillation frequency was stabilized for 10 min under $\mathrm{N}_{2}$ gas atmosphere in the container at a flow rate of $0.5 \mathrm{ml} / \mathrm{min}$. After the oscillation frequency of the QCM was stabilized, the nitrogen gas flow was stopped and the sample gas to be adsorbed was introduced for $20 \mathrm{~min}$ at a flow rate of $0.2 \mathrm{ml} / \mathrm{min}$. Sample gases were prepared using a gas generator, Permeater PD-1B (GASTEC Corporation, Kanagawa, Japan), that uses a diffusion tube. When the frequency of the QCM became constant after $20 \mathrm{~min}$, the sample gas flow was stopped, and $\mathrm{N}_{2}$ gas was introduced again as

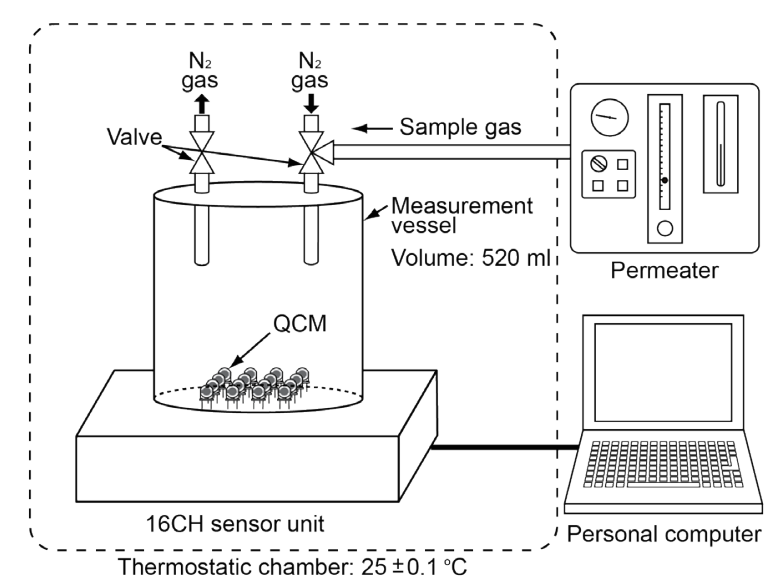

Fig. 2. Schematic diagram of 16-channel QCM array for gas sorption measurement. 
desorption gas. In each sample gas as well as in each pp film, the shifts in frequency due to gas adsorption were measured three times under the same operation conditions.

\section{Results and Discussion}

\section{1 pp film}

The thicknesses of pp-CMS and pp-VBA films at RF powers of 90 to $150 \mathrm{~W}$ are shown by closed circles and squares in Fig. 3, respectively. The coating amounts of the pp film were calculated from the amount of frequency shift of the QCM. We have reported that pp film thickness changes with RF power, ${ }^{(19-21)}$ but it was clarified that pp-CMS has low dependence on RF power in the range from 90 to $150 \mathrm{~W}$. Thus far, when the pressure of monomer supply into the bel jar is $100 \mathrm{~Pa}$, it has been reported that the increase in RF power promotes polymerization by increasing the energy during polymerization and changes the pp film thickness. However, the supply pressure of the CMS monomer used this time was $5 \mathrm{~Pa}$, which is the condition found to supply the monomer at a constant flow rate because the amount of CMS monomer vaporized is small. Under these conditions, the deposition level is constant, but it was found that the amount of pp-CMS deposited does not depend on RF power, unlike the conventional polymerization method. By contrast, the pp-VBA film became 10 times thicker than the ppCMS film, presumably because the monomer was introduced by sublimation, in which the amount of monomer supplied is difficult to control.

\subsection{Chemical structure analysis and surface morphology observation of pp films}

Figures 4(a) and 4(b) respectively show the FT-IR spectra of pp-CMS and pp-VBA coated on $\mathrm{KBr}$ tablets at RF powers of 90 to $150 \mathrm{~W}$. In the IR spectra of pp-CMS shown in Fig. 4(a), $\mathrm{C}=\mathrm{C}$ stretching $\left(\mathrm{A}, 1707 \mathrm{~cm}^{-1}\right)$ and $\mathrm{C}-\mathrm{H}$ bending $\left(\mathrm{B}, 1431 \mathrm{~cm}^{-1}\right)$ caused by aromatic rings were observed. Considering that $674 \mathrm{~cm}^{-1}$ of $\mathrm{C}$ shows $\mathrm{C}-\mathrm{Cl}$ stretching, pp-CMS is considered to be polymerized while maintaining the structures of the $\mathrm{Cl}$ group and aromatic ring. By contrast,

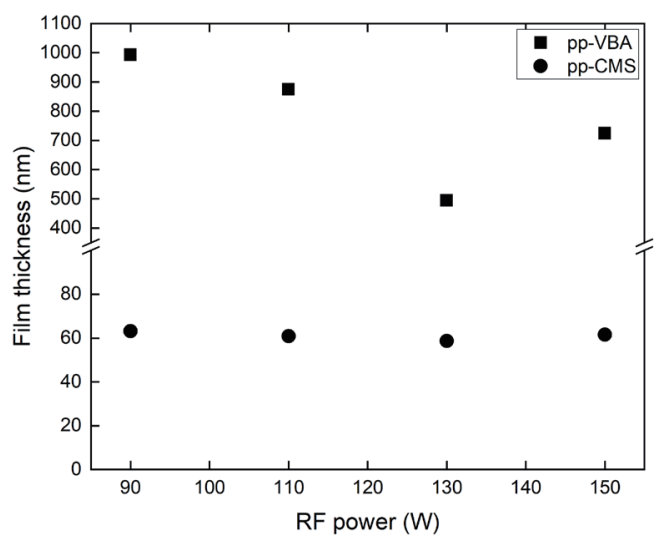

Fig. 3. Film thickness depending on RF power of pp-CMS and pp-VBA. 


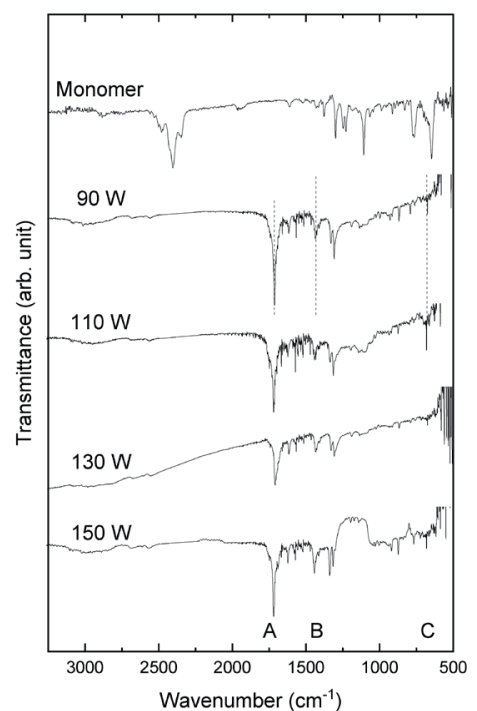

(a)

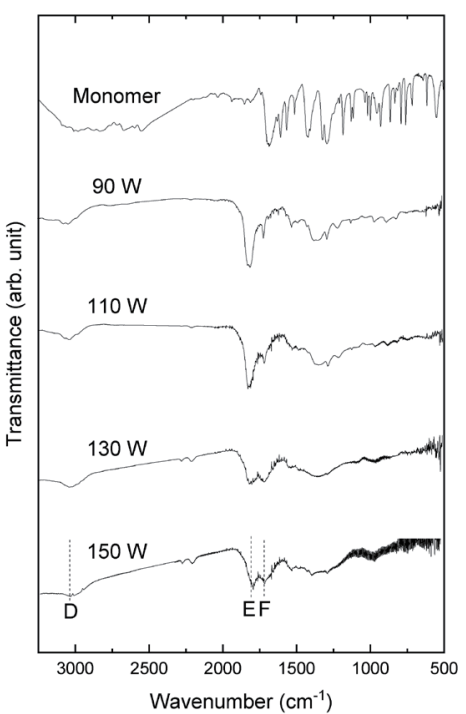

(b)

Fig. 4. FT-IR spectra of (a) pp-CMS and (b) pp-VBA at each RF power.

the IR spectrum of pp-VBA shown in Fig. 4(b) shows OH stretching (D, $3032 \mathrm{~cm}^{-1}$ ) caused by $\mathrm{COOH}$ groups, and also $\mathrm{C}=\mathrm{O}$ stretching $\left(\mathrm{E}, 1800 \mathrm{~cm}^{-1}\right)$ and $\mathrm{C}=\mathrm{C}$ stretching $\left(\mathrm{F}, 1717 \mathrm{~cm}^{-1}\right)$ were confirmed. From these IR spectral analysis results, it was found that pp-VBA maintained the structures of the aromatic ring and $\mathrm{COOH}$ groups even during the polymerization at each $\mathrm{RF}$ power.

Figure 5 shows the SEM micrographs of pp-CMS and pp-VBA coated on QCMs at RF powers of 90 to $150 \mathrm{~W}$. SEM observation confirmed that pp-CMS formed a smooth thin film at each RF power. pp-VBA was confirmed to be smooth when the RF power was $90 \mathrm{~W}$, but the roughness of the film surface increased as RF power increased to 110,130 , and $150 \mathrm{~W}$. This is considered to be due to the fact that the polymerization of the monomer sublimated in the plasma space was promoted with the increase in RF power, and the film was deposited in an island morphology.

\subsection{Sample gas adsorption and RS analysis}

Since the pp film thickness varies depending on the monomer and RF power, the amount of frequency change due to gas adsorption varies even with the same chemical structure. The amount of frequency change due to gas adsorption also varies depending on the molecular weight and concentration of the sample gas. Therefore, the adsorption sensitivity of each pp film and sample gas was evaluated by standardizing the RS to $1 \mathrm{ppm}$ sample gas. After measuring adsorption amounts of six sample gases (ethanol, acetone, methanol, 2-propanol, dichloromethane, and chloroform) with different pp-CMS and pp-VBA films coated on QCMs at RF powers of $90,110,130$, and $150 \mathrm{~W}, \mathrm{RS}$ to $1 \mathrm{ppm}$ sample gas was calculated as ${ }^{(17,23)}$ 


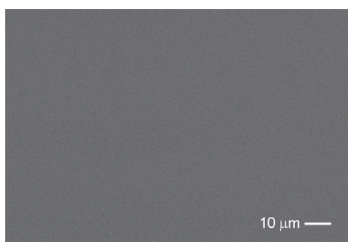

(a)

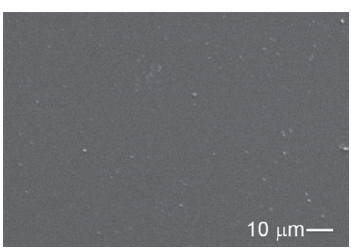

(e)

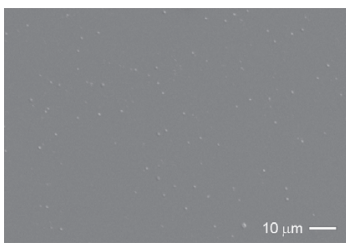

(b)

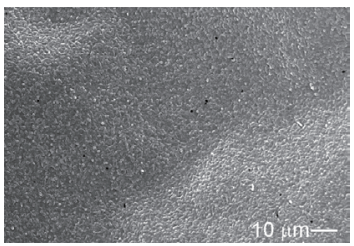

(f)

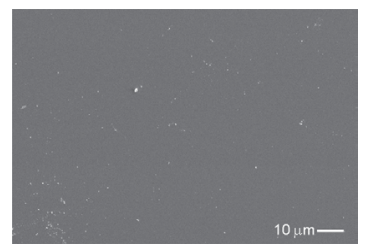

(c)

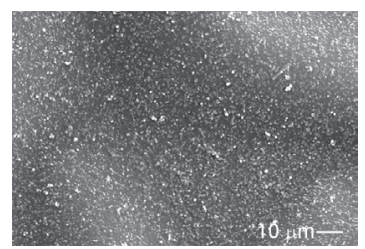

$(\mathrm{g})$

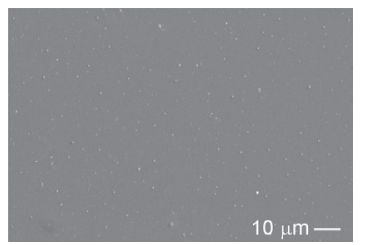

(d)

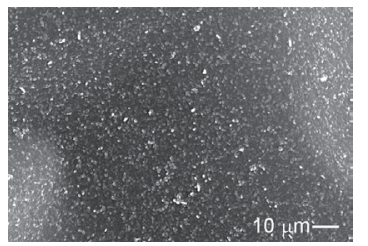

(h)

Fig. 5. SEM micrographs of surfaces of QCM devices coated with pp films. (a) pp-CMS at $90 \mathrm{~W}$, (b) pp-CMS at $110 \mathrm{~W}$, (c) pp-CMS at $130 \mathrm{~W}$, (d) pp-CMS at $150 \mathrm{~W}$, (e) pp-VBA at $90 \mathrm{~W}$, (f) pp-VBA at $110 \mathrm{~W}$, (g) pp-VBA at $130 \mathrm{~W}$, and (h) pp-VBA at $150 \mathrm{~W}$.

$$
R S=\frac{G / M_{1}}{P / M_{0}} \times \frac{1}{C}
$$

where $R S$ is the $R S$ to the sample gas $\left(\mathrm{ppm}^{-1}\right), P$ is the amount of pp film coated on the QCM (ng), $M_{0}$ is the molecular weight of the monomer $(\mathrm{g} / \mathrm{mol}), G$ is the sorption amount of the sample gas (ng), $M_{1}$ is the molecular weight of the sample gas, and $C$ is the concentration of the sample gas (ppm). The $C$ of each sample gas was measured using a gas detector tube (GASTEC Corporation, Kanagawa, Japan). The concentrations of ethanol, acetone, methanol, 2-propanol, dichloromethane, and chloroform were determined to be 330, 1269, 950, 207, 1344, and 865 ppm, respectively.

QCM measurements are known to be affected by humidity. ${ }^{(24,25)}$ The gas sorption measurement of each pp film was performed in a thermostatic chamber at a temperature of $25{ }^{\circ} \mathrm{C}$ and a humidity of about 50 to $60 \%$. Although the effect of humidity alone on each pp film has not been evaluated, the selectivity to the target gas by each pp film is considered to be high even under conditions where the humidity ranges from 50 to $60 \%$.

The RSs of pp-CMS and pp-VBA coated on QCMs to each sample gas are shown in Figs. 6(a) and 6(b), respectively. Both pp-CMS and pp-VBA exhibited high RSs to ethanol, methanol, and 2-propanol containing hydroxy groups, possibly because the amount of gas adsorbed by hydrogen bonding increased. Also, pp-CMS showed a higher RS to dichloromethane and chloroform than pp-VBA. This is presumably because the molecule of the pp-CMS film contained the same chloro groups as the VOCs. By contrast, as clarified by FT-IR analysis, ppVBA facilitated the adsorption of alcohols such as ethanol, 2-propanol, and methanol owing to the presence of carboxy groups within the molecule of the pp-VBA film, whose structure is different from that of the pp-CMS film. In particular, the adsorption of methanol is considered to be promoted because of methanol has the lowest molecular weight among the three alcohols.

Figure 7 shows the RSs of four pp films ${ }^{(20)}$ containing aromatic ring structures to (a) dichloromethane and (b) methanol. The comparison among these four pp membranes revealed 


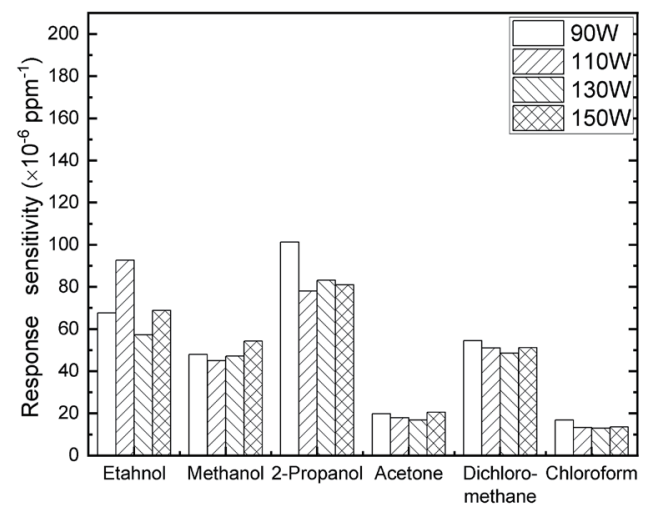

(a)

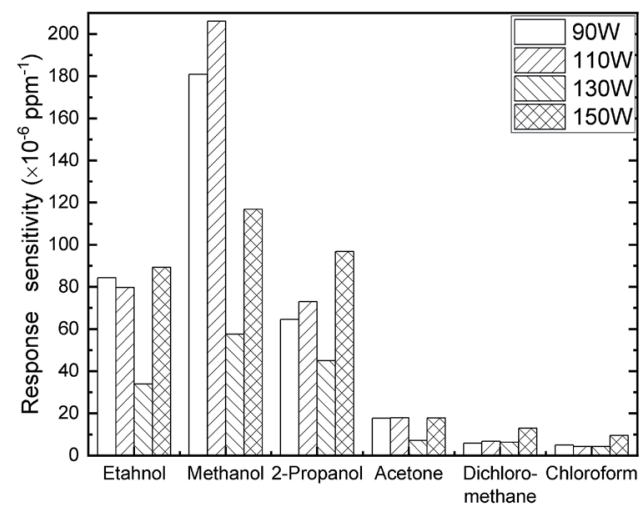

(b)

Fig. 6. RSs of (a) pp-CMS and (b) pp-VBA coated on QCM to sample gases.

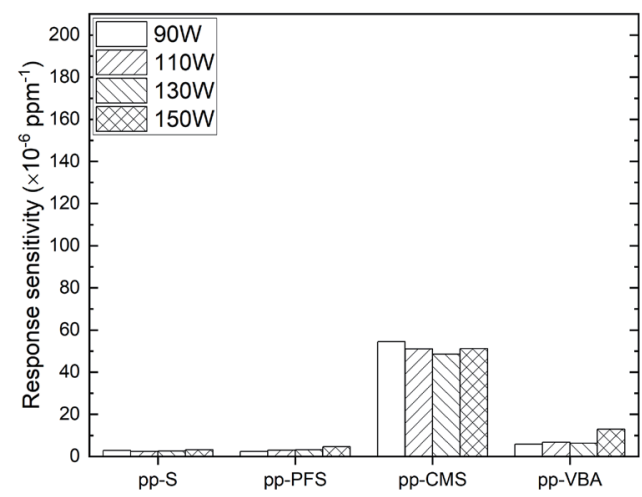

(a)

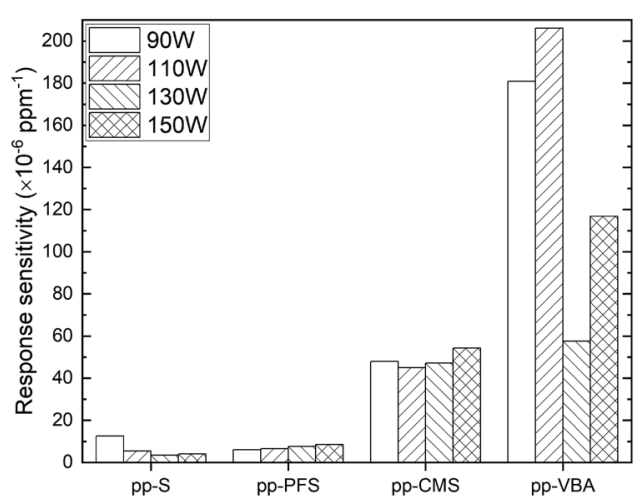

(b)

Fig. 7. RSs of pp-S (styrene), pp-PFS (2,3,4,5,6-pentafluorostyrene), pp-CMS [4-(chloromethyl) styrene], and ppVBA (4-vinylbenzoic acid) coated on QCM to (a) dichloromethane and (b) methanol.

that the pp-CMS membrane showed the highest RS to dichloromethane. This increase in RS may be due to the increased level of adsorption between the chloro groups in pp-CMS and dichloromethane, but further analysis is required to clarify its mechanism.

\section{Conclusions}

In this study, CMS and VBA coatings were applied to QCMs by plasma polymerization in order to develop an organic gas sensor using QCMs. The resultant pp films polymerized at each RF power were analyzed for their chemical structures by FT-IR. The RSs to six sample gases were evaluated, and it was revealed that both pp-CMS and pp-VBA have high RSs to ethanol, methanol, and 2-propanol. Furthermore, it was found that pp-CMS has specific RS to dichloromethane and pp-VBA to methanol. 


\section{Acknowledgments}

This study was financially supported by AIST.

\section{References}

1 Y. Nezu, R. Hayashi and Y. Oshima: J. Environ. Safe. 6 (2015) 99. https://doi.org/10.11162/daikankyo. E14PROCP14

2 C. A. Amorim and Z. L. Cardeal: J. Chromatogr. B 853 (2007) 1. https:doi.org/10.1016/j.jchromb.2007.03.023

3 S. H. Zhang, J. P. You, C. Kennes, Z. W. Cheng, J. X. Ye, D. Z. Chen, J. M. Chen, and L. D. Wang: Chem. Eng. J. 334 (2018) 2625. https://doi.org/10.1016/j.cej.2017.11.014

4 B. B. Palm, J. P. Campuzano, A. M. Ortega, D. A. Day, L. Kaser, W. Jud, T. Karl, A. Hansel, J. F. Hunter, E. S. Cross, J. H. Kroll, Z. Peng, W. H. Brune, and J. L. Jimenez: Atmos. Chem. Phys. 16 (2016) 2943. https://doi. org/10.5194/acp-16-2943-2016

5 A. Y. Bigazzi, M. A. Figliozzi, W. Luo, and J. F. Pankow: Environ. Sci. Technol. 50 (2016) 5357. https://doi. org/10.1021/acs.est.6b01159

6 A. S. Park, B. Ritz, C. Ling, M. Cockburn, and J. E. Heck: Int. J. Hyg. Environ. Health. 220 (2017) 1133. http:// dx.doi.org/10.1016/j.ijheh.2017.06.006

7 K. Koyama, S. Kubo, A. Ueki, T. Shimono, S. Takemura, S. Tanaka, M. Kinoshita, G. Hamano, and Y. Miki: Jpn. J. Radiol. 35 (2017) 233. https://doi.org/10.1007/s11604-017-0626-y

8 A.M. Nabola, B. M. Broderick, and L. W. Gill: Atmos. Environ. 42 (2008) 6496. https://doi.org/10.1016/ j.atmosenv.2008.04.015

9 L. I. Panis, B. D. Geusc, G. Vandenbulcke, H. Willems, B. Degraeuwe, N. Bleux, V. Mishra, I. Thomas, and R. Meeusen: Atmos. Environ. 44 (2010) 2263. https://doi.org/10.1016/j.atmosenv.2010.04.028

10 R. H. Ramm, M. Jakubowski, B. Heinzow, J. M. Christensen, E. Olsen, and O. Hertel: Pure Appl. Chem. 72 (2000) 385. http://dx.doi.org/10.1351/pac200072030385

11 J. F Pankow, W. Luo, D. A. Bender, L. M. Isabelle, J. S Hollingsworth, C. Chen, W. E Asher, and J. S. Zogorski: Atmos. Environ. 37 (2003) 5023. https://doi.org/10.1016/j.atmosenv.2003.08.006

12 A. W Boots, J. J. B. N. Berkel, J. W. Dallinga, A. Smolinska, E. F. Wouters and F. J. Schooten: J. Breath Res. 6 (2012) 027108. http://doi.org/10.1088/1752-7155/6/2/027108

13 C. Lu and A.W. Czanderna: Applications of Piezoelectric Quartz Crystal Microbalances (Elsevier Science Publishers, Amsterdam, 1984). https://www.elsevier.com/books/applications-of-piezoelectric-quartz-crystalmicrobalances/lu/978-0-444-42277-4

14 G. Sauerbrey: Zeitschrift fur Physik 155 (1959) 206. https://doi.org/10.1007/BF01337937

15 S. Kurosawa, J. W. Park, H. Aizawa, S. Wakida, H. Tao, and K. Ishihara: Biosens. Bioelectron. 22 (2006) 473. https://doi.org/10.1016/j.bios.2006.06.030

16 H. Yasuda: Plasma Polymerization (Academic Press, New York, 1985) Chaps. 1, 2, and 6. https://www. elsevier.com/books/plasma-polymerization/yasuda/978-0-12-768760-5

17 S. Kurosawa, H. Miura, H. Takahashi, J. W. Park, H. Aizawa, K. Noda, K. Yamada and M. Hirata: Sens. Actuators, B 108 (2005) 558. https://doi.org/10.1016/j.snb.2004.12.086

18 H. Muguruma: Trends Anal. Chem. 26 (2007) 433. https://doi.org/10.1016/j.trac.2007.03.003

19 H. Aizawa, S. Kawashima, S. Kurosawa, K. Noda, T. Fujii, and M. Hirata: Thin Solid Films 515 (2007) 4141. https://doi.org/10.1016/j.tsf.2006.02.047

20 S. Kurosawa, T. Hirokawa, K. Kashima, H. Aizawa, D. S. Han, Y. Yoshimi, Y. Okada, K. Yase, J. Miyake, M. Yoshimoto, and J. Hilborn: Thin Solid Films 374 (2000) 262. https://doi.org/10.1016/S0040-6090(00)01161-5

21 S. Kurosawa, B. Atthoff, H. Aizawa, and J. Hilborn: Thin Solid Films 457 (2004) 26. https://doi.org/10.1016/ j.tsf.2003.12.008

22 S. Kurosawa, B. G. Choi, J. W. Park, H. Aizawa, K. B. Shim, and K. Yamamoto: Thin Solid Films 506 (2006) 176. https://doi.org/10.1016/j.tsf.2005.08.210

23 H. Aizawa, T. Muramatsu, K. Noda, R. Naganawa, S. Kurosawa, R. Tsuruyama and S. Sunohara: J. Photopolym Sci. Technol. 24 (2011) 459. https://doi.org/10.2494/photopolymer.24.459

24 B. Mumyakmaz, A. Özmen, M. A. Ebeoğlua, C. Taşaltin, and İ. Gürol: Sens. Actuators, B 147 (2010) 277. https://doi.org/10.1016/j.snb.2010.03.019

25 S. Korposh, R. Selyanchyn, and S. W. Lee: Sens. Actuators, B 147 (2010) 599. https://doi.org/10.1016/ j.snb.2010.04.006 